\title{
Hepatic injury is associated with cell cycle arrest and apoptosis with alteration of cyclin A and D1 in ammonium chloride-induced hyperammonemic rats
}

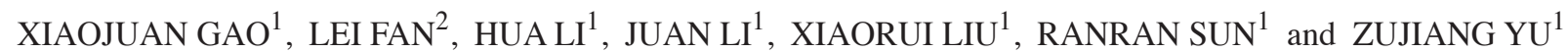 \\ ${ }^{1}$ Department of Infectious Diseases, The First Affiliated Hospital of Zhengzhou University, Zhengzhou, Henan 450052; \\ ${ }^{2}$ Department of Pharmacy, Children's Hospital of Zhengzhou City, Zhengzhou, Henan 450053, P.R. China
}

Received February 8, 2015; Accepted November 5, 2015

DOI: 10.3892/etm.2015.2931

\begin{abstract}
Hyperammonemia is considered to be central to the pathophysiology of hepatic encephalopathy in patients exhibiting hepatic failure (HF). It has previously been determined that hyperammonemia is a serious metabolic disorder commonly observed in patients with HF. However, it is unclear whether hyperammonemia has a direct adverse effect on hepatic cells or serves as a cause and effect of HF. The present study investigated whether hepatic injury is caused by hyperammonemia, and aimed to provide an insight into the causes and mechanisms of HF. Hyperammonemic rats were established via intragastric administration of ammonium chloride solution. Hepatic tissues were assessed using biochemistry, histology, immunohistochemistry, flow cytometry (FCM), semi-quantitative reverse transcription-polymerase chain reaction and western blot analysis. Hyperammonemic rats exhibited significantly increased levels of liver function markers, including alanine transaminase $(\mathrm{P}<0.01)$, aspartate aminotransferase $(\mathrm{P}<0.01)$, blood ammonia $(\mathrm{P}<0.01)$ and direct bilirubin $(\mathrm{P}<0.05)$, which indicated hepatic injury. A pathological assessment revealed mild hydropic degeneration, but no necrosis or inflammatory cell infiltration. However, terminal deoxynucleotidyl transferase dUTP nick end-labeling assays confirmed a significant increase in the rate of cellular apoptosis in hyperammonemic rat livers $(\mathrm{P}<0.01)$. FCM analysis revealed that there were significantly more cells in the $S$ phase and fewer in the $G_{2} / M$ phase $(P<0.01)$, and the expression levels of cyclin A and D1 mRNA and proteins were significantly increased $(\mathrm{P}<0.01)$. In summary, cell cycle arrest, apoptosis and an alteration of cyclin A and D1 levels were all markers of hyperammonemia-induced hepatic injury. These findings provide an insight into the potential mechanisms
\end{abstract}

Correspondence to: Professor Zujiang Yu, Department of Infectious Diseases, The First Affiliated Hospital of Zhengzhou University, 40 Daxue Road, Zhengzhou, Henan 450052, P.R. China E-mail: johnyuem@zzu.edu.cn; fanlei202@126.com

Key words: hyperammonemia, cell-cycle arrest/apoptosis, cyclin A, cyclin D1, hepatic injury underlying hyperammonemia-induced hepatic injury, and may be used as potential targets for treating or preventing hepatic damage caused by hyperammonemia, including hepatic encephalopathy.

\section{Introduction}

Ammonia is produced in the gastrointestinal tract in two ways: Colonic bacteria produce ammonia through the catabolism of nitrogenous waste from dietary protein intake, and skeletal muscle degrades and produces ammonia during seizures and intense exercise (1). Ammonia is primarily hepatically eliminated through the urea cycle, during which it is converted to glutamine and renally excreted (2). Normal blood ammonia (BA) is $35 \mathrm{mmol} / \mathrm{l}$, and any increase in this concentration is typically due to its increased production or decreased elimination.

Hepatic failure (HF), which is a serious disease with a high mortality rate $(70-80 \%)$, is predominantly caused by hepatitis $\mathrm{B}$ and $\mathrm{C}$ viral infections $(3,4)$. Existing treatment strategies for hepatic failure include artificial liver supporting systems and liver transplantation (5). During HF, normal metabolism of ammonia is blocked, which leads to an accumulation of ammonia in the circulation. This results in an increase in hepatocyte necrosis and apoptosis (6-8). However, it is uncertain if hyperammonemia adversely affects residual hepatocyte function in HF patients, which would cause a 'vicious cycle' by further aggravating the hepatic injury (9). In previous studies, a 'two-hit theory' was used to administer antiviral therapy and liver function maintenance using 1-ornithine-1-aspartate to slow progression of hepatitis B virus-related acute-on-chronic HF (10-13). Therefore, the present study hypothesized that hepatic injury induced by hyperammonemia may be activated via apoptotic signaling pathways. In order to determine this, a rat model of hyperammonemia was established using intragastric administration of ammonium chloride solution, according to a previous study (14), and cell proliferation and apoptosis of rat hepatic cells were observed.

\section{Materials and methods}

Animals, experimental design and specimen collection. Male Sprague-Dawley (SD) rats $(n=24$; weight, $200 \pm 25 \mathrm{~g}$; 
age, 6 weeks) were obtained from the Animal Experiment Center of the Henan Province (Zhengzhou, China). The rats were maintained at $22 \pm 2^{\circ} \mathrm{C}$ under a 12 -h light/dark cycle, with ad libitum access to water. The rats were fed experimental particle feed provided by the Animal Experimental Center of the Henan Province. The animal protocol was approved by the Animal Care and Use Committee of the Zhengzhou University (Zhengzhou, China; approval no. ZZ-83042-9). Animals were cared for according to the institutional guidelines of Zhengzhou University.

Experimental groups. The SD rats $(\mathrm{n}=24)$ were randomly divided into two groups: i) The hyperammonemic group, in which hyperammonemia was induced by treating the rats with $10 \%$ ammonium chloride solution $(10 \mathrm{ml} / \mathrm{kg}$; Sangon Biotech Co., Ltd., Shanghai, China) via intragastric administration twice daily for 30 days; and ii) the control group, in which the rats were treated with saline $(10 \mathrm{ml} / \mathrm{kg})$ via the same route of administration and dosing regimen as the hyperammonemic group. After 30 days, the rats were sacrificed by cervical dislocation and blood and liver tissue samples were collected, according to methods outlined in previous studies $(14,15)$.

Evaluating serum alanine transaminase (ALT), aspartate transaminase (AST), direct bilirubin (DBil) and blood ammonia (BA) concentration levels. Serum ALT, AST and DBil concentration levels were assayed using the ALT Assay, AST Assay and Total Bilirubin enzyme-linked immunosorbent assay kits obtained from Suzhou Calvin Biotechnology (Suzhou, China). BA was measured using a standard clinical automatic analyzer (AA-4120 Ammonia Checker II; Kyoto Daiichi Kagaku Co., Ltd., Kyoto, Japan).

Histological analysis. Hepatic tissue blocks obtained from the rats in all groups were dehydrated, embedded in paraffin, sectioned $(5 \mu \mathrm{m})$ and stained with hematoxylin and eosin (Nanjing Jiancheng Bioengineering Institute, Nanjing, China). The histological analysis of the tissue sections was conducted by two independent pathologists who were blind to the study design and the specimen identities. Sections were observed under a light microscope (LSM-510; Carl Zeiss AG, Oberkochen, Germany).

Assessment of apoptosis. To quantify apoptosis, a terminal deoxynucleotidyl transferasedUTPnick end-labeling (TUNEL) assay was performed on paraffin-embedded tissue sections from each rat (16). Hepatic tissue sections were subjected to a TUNEL assay, and analyzed with an In Situ Cell Death Detection kit, POD (Roche Diagnostics, Shanghai, China). Briefly, hepatic tissue sections were dewaxed, rehydrated and then incubated with $50 \mu \mathrm{l}$ TUNEL reaction mixture at room temperature in the dark for $1 \mathrm{~h}$. After washing three times with phosphate-buffered saline (PBS), the tissue sections were counterstained with $0.0002 \%$ 4',6-diamidino-2-phenylindole (Sangon Biotech, Co., Ltd.). Positive cells were scored under the Nikon Eclipse Ti-U fluorescence microscope (Nikon Corporation, Tokyo, Japan). This was performed by counting cells under low magnification (x100) in 10 separate arbitrary fields in each tissue section. Cells with a brown nuclei were interpreted as being positively apoptotic. Student's t-tests were used to calculate any statistical significance. The apoptotic index was expressed as a percentage of the TUNEL-positive cells.

Cell cycle analysis. Cells were collected from hepatic tissue in a humidified chamber at room temperature, and the cell concentration was adjusted to $1 \times 10^{6}$ cells $/ \mathrm{ml}$. Cells were washed with cold PBS and fixed in 70\% cold ethanol (Tianjin Siyou Science and Technology Development Co., Ltd., Tianjin, China) overnight at $4^{\circ} \mathrm{C}$. A fluorochrome solution (Sangon Biotech, Co., Ltd.), containing $3.4 \mathrm{mmol} / \mathrm{l}$ sodium citrate ion, $1 \%$ Triton X-100, $20 \mu \mathrm{g} / \mathrm{ml}$ RNase A and $50 \mu \mathrm{g} / \mathrm{ml}$ propidium iodide, was added and the mixture was incubated in the dark at room temperature for $0.5 \mathrm{~h}$ (17). Cell cycle distribution was measured using flow cytometry (FCM; Sysmex Partec GmbH, Münster, Germany). FCM analysis was performed using CellQuest software, version 3.0 (BD Biosciences, Franklin Lakes, NJ, USA).

Immunohistochemistry (IHC). To measure hepatic cyclin A and D1 expression, a standard IHC protocol was followed to stain the rat liver tissue samples (18). Briefly, 5- $\mu \mathrm{m}$ paraffin-embedded tissue samples were de-paraffinized with xylene (Sangon Biotech, Co., Ltd.), and endogenous peroxidase activity was quenched with $3 \% \mathrm{H}_{2} \mathrm{O}_{2}$ in methanol (Sangon Biotech, Co., Ltd.) for $30 \mathrm{~min}$ in the dark. Tissue samples were dehydrated through an alcohol gradient and subjected to antigen retrieval using $10 \mathrm{mM}$ sodium citrate (Sangon Biotech, Co., Ltd.). Rabbit anti-rat cyclin A (1:500; sc-751), cyclin D1 $(1: 500 ;$ sc-753) and $\beta$-actin $(1: 5,000 ;$ sc-130656) polyclonal antibodies (all Santa Cruz Biotechnology, Inc., Dallas, TX, USA) were used as a primary antibodies. Sections were then treated with a goat anti-rabbit biotin-conjugated secondary antibody (1:1,000; sc-2004; Santa Cruz Biotechnology, Inc.) followed by streptavidin (Sangon Biotech, Co., Ltd.). After washing three times with PBS, the tissue sections were incubated with 3,3'-diaminobenzidine tetrahydrochloride (Sigma-Aldrich, St-Louis, MO, USA) for $30 \mathrm{~min}$, and immediately washed under tap water following color development. Slides were then counterstained with hematoxylin. Slides were mounted with dibutyl phthalate xylene and were then observed under a light microscope (LSM-510; Carl Zeiss AG). The status of nuclear expression of cyclin A and D1 were determined using integrated optical density, as measured with Biosens Digital Imaging System software, version 1.6 (Shanghai Biotechnology Corporation, Shanghai, China).

Semi-quantitative reverse transcription-polymerase chain reaction $(R T-P C R)$ assay. Three preliminary experiments were conducted prior to semi-quantitative RT-PCR, in order to validate the linearity of PCR product accumulation with increasing number of PCR cycles. Briefly, aliquots containing fixed amounts of cDNA mixture were subjected to amplification for varying numbers of PCR cycles (between 20 and 40 cycles), using the S1000 ${ }^{\mathrm{TM}}$ Thermal Cycler (Bio-Rad Laboratories Co., Ltd, California, USA). Total RNA was extracted from liver tissues using TRIzol ${ }^{\circledR}$ reagent (Thermo Fisher Scientific, Inc., Waltham, MA, USA), according to the manufacturer's protocol. Purified RNA treated with DNAase (TURBO DNA-free ${ }^{\text {TM }}$ kit; Thermo Fisher Scientific, Inc.) 
A

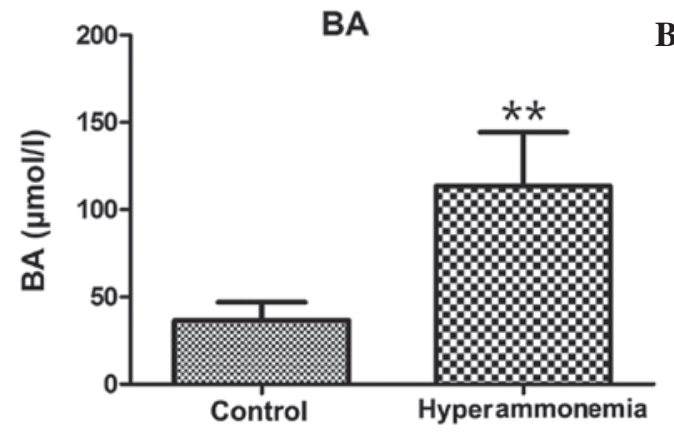

C

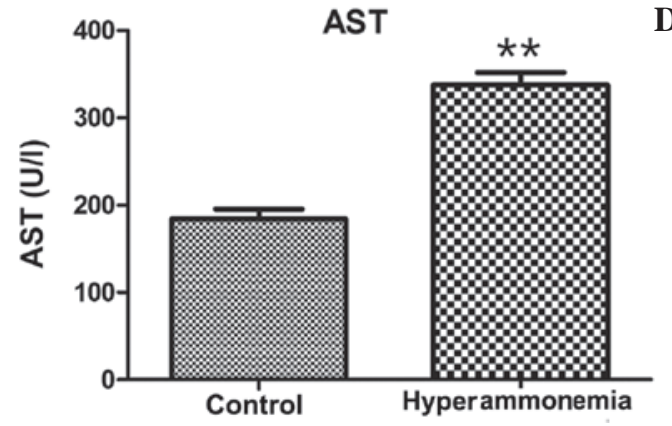

B

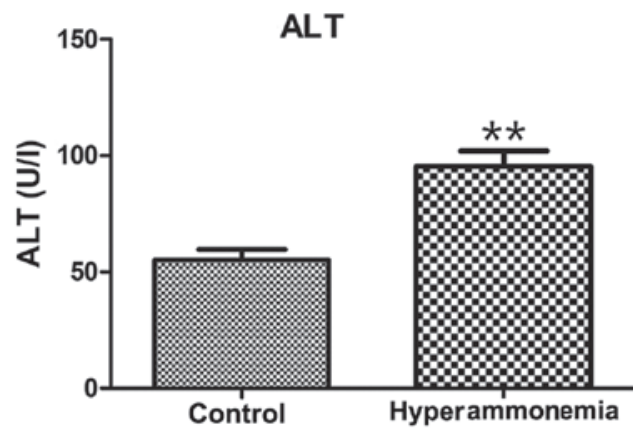

D

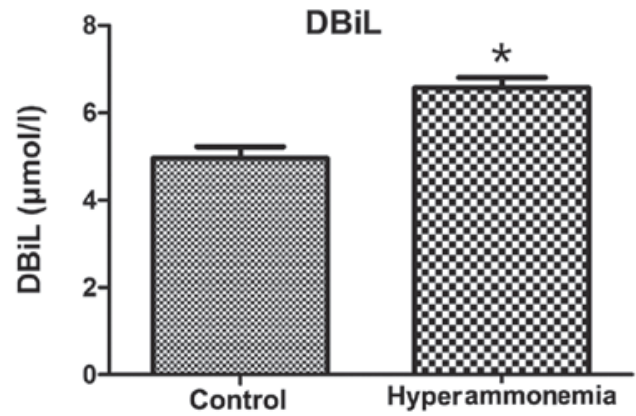

Figure 1. Biochemical analysis of rat blood samples, collected after the repeat administration of $10 \%$ ammonium chloride. The levels of (A) BA, (B) ALT, (C) AST and (D) DBil were measured. "P $<0.05$ and ${ }^{* *} \mathrm{P}<0.01$ vs. the control. Data are presented as the mean \pm standard deviation. BA, blood ammonia; ALT, alanine transaminase; AST, aspartate transaminase; DBil, direct bilirubin.

A

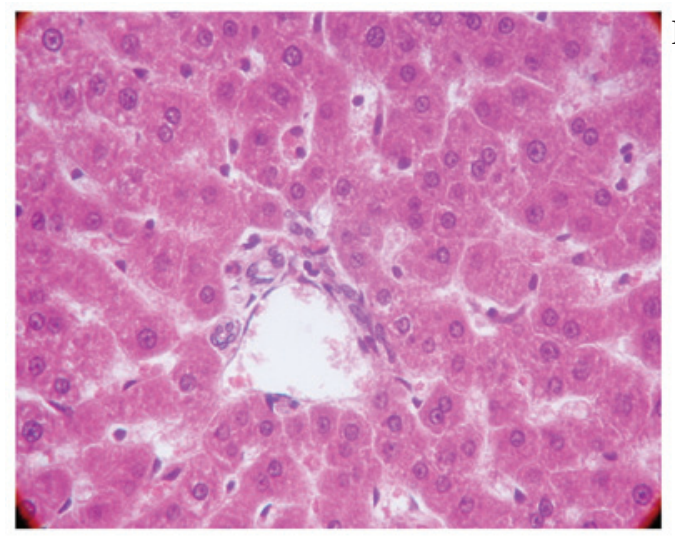

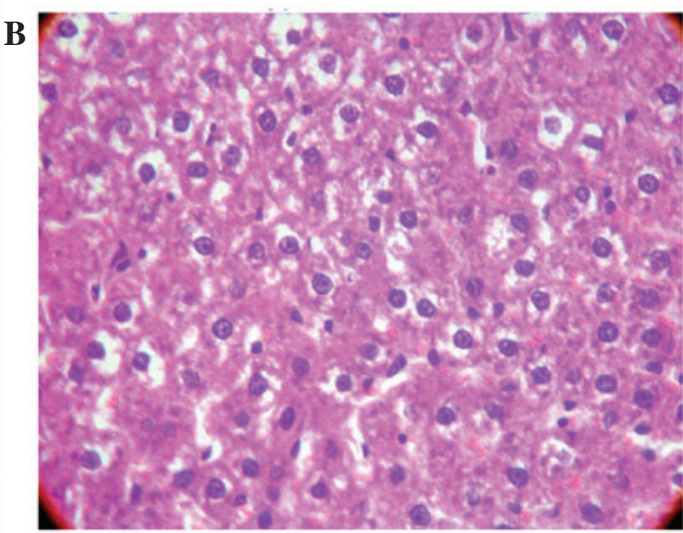

Figure 2. Hepatic histopathology in rats subjected to hyperammonemia. Light photomicrographs of liver sections were obtained from the (A) control and (B) hyperammonemic rats. Hematoxylin and eosin staining; magnification, $\mathrm{x} 400$.

was reverse-transcribed into cDNA using random hexamers (Sangon Biotech, Co., Ltd.) and the SuperScript First-Strand Synthesis System for RT-PCR kit (Thermo Fisher Scientific, Inc.). PCR was conducted using the $S 1000^{\mathrm{TM}}$ Thermal Cycler and the following cycling conditions: $90^{\circ} \mathrm{C}$ denaturation for $1 \mathrm{~min}$, followed by 30 cycles of denaturation at $90^{\circ} \mathrm{C}$ for $60 \mathrm{sec}$, annealing at $60^{\circ} \mathrm{C}$ for $60 \mathrm{sec}$ and extension at $72^{\circ} \mathrm{C}$ for $60 \mathrm{sec}$, and a final extension step of $72^{\circ} \mathrm{C}$ for $10 \mathrm{~min}$. The PCR primers were as follows: Sense, 5'-CAAAGTGTG CCGTTGTCTCTT-3' and antisense, 5'-ATCTGCGCTTGG AGTGATAGA-3' for cyclin A; sense, 5'-CCACGATTTCAT CGAACACTT-3' and antisense for cyclin D1, 5'-CTCTGG AAAGAAAGTGCGTTG-3'; and sense, 5'-CAGTGCCAG CCTCGTCTCAT-3' and antisense, 5'-AGGGGCCATCCA
CAGTCTTC-3' for glyceraldehyde-3-phosphate dehydrogenase (Sangon Biotech, Co., Ltd.). A negative control and an RT-minus control were used in order to verify the results of the first strand cDNA synthesis step. Experiments were performed in triplicate. Prior to amplification of each gene, normalization was carried out with the endogenous control gene, GADPH. All PCR products and the 100 bp DNA ladder (cat no. MD109; Tiangen Biotech Co., Ltd., Beijing, China) were separated by $2 \%$ agarose gel electrophoresis (Sangon Biotech, Co., Ltd.). The amplified DNA bands on the agarose gels were detected using ethidium bromide staining (Sangon Biotech, Co., Ltd.) and were quantified using the ImageQuant ${ }^{\mathrm{TM}}$ LAS 500 CCD imager (GE Healthcare Life Sciences, Chalfont, UK). 
A
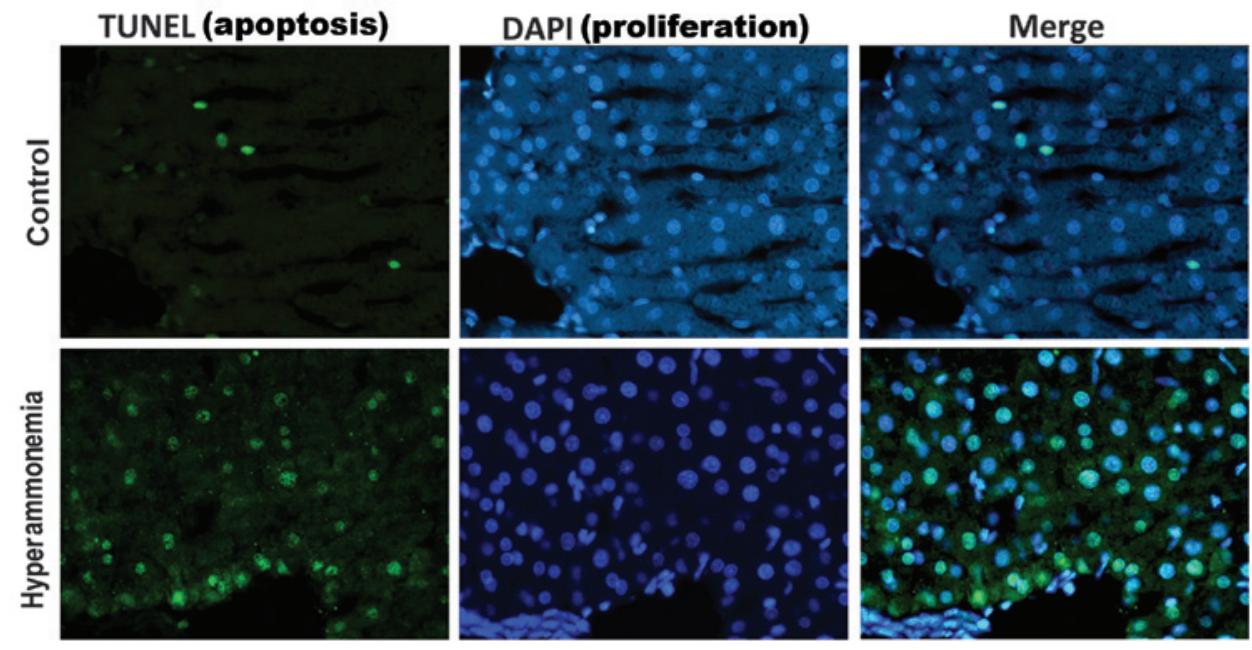

B

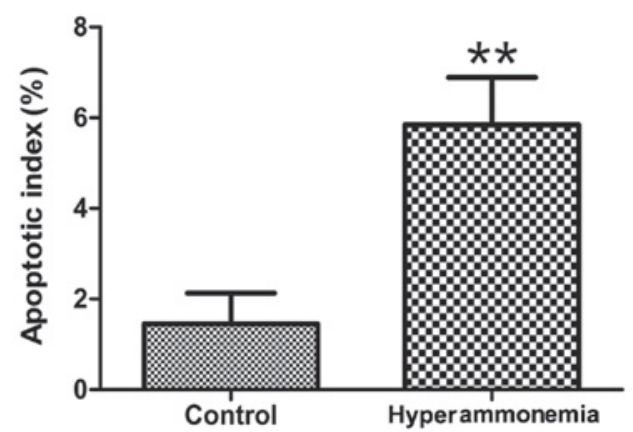

Figure 3. Hyperammonemia-induced hepatic cell apoptosis. (A) A TUNEL assay revealed apoptotic-positive hepatic cells marked by green staining. The blue DAPI stain marks intact DNA. Magnification, $\mathrm{x} 400$. (B) The apoptotic index. ${ }^{* *} \mathrm{P}<0.01$ vs. the control. Data are presented as the mean \pm standard deviation. TUNEL, terminal deoxynucleotidyl transferase dUTP nick end-labeling.

Preparation of lysates and western blotting. Hepatic tissues were frozen on dry ice upon harvesting. Homogenates were sonicated in lysis buffer [150 mM NaCl, $1.0 \%$ IGEPAL,

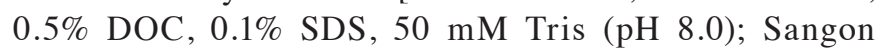
Biotech, Co., Ltd.] and centrifuged (Heraeus Biofuge Stratos Centrifuge; Thermo Fisher Scientific, Inc.) at 21,890 x g and a temperature of $4^{\circ} \mathrm{C}$ for $30 \mathrm{~min}$. Supernatants were boiled in Laemmli sample buffer (Santa Cruz Biotechnology, Inc.) for western blotting at room temperature. The antibodies used were as follows: Anti-cyclin D1 (cat no. H-295; dilution, 1:1,000; Santa Cruz Biotechnology, Inc.) and anti-cyclin A (cat no. H-432; dilution, 1:1,000; Santa Cruz Biotechnology, Inc.). Western blots were quantified using ImageJ software, version 1.48 (National Institutes of Health, Bethesda, MD, USA).

Statistical analysis. Statistical analyses were conducted using SPSS software, version 19.0 (IBM SPSS, Armonk, NY, USA). Data are depicted as the mean \pm standard deviation. Student's t-test was used to examine the statistical significance between groups. $\mathrm{P}<0.05$ was considered to indicate a statistically significant difference.

\section{Results}

Hyperammonemia induces an increase in the concentrations of $B A, A L T, A S T$ and DBil. The concentrations of BA, ALT, AST and DBit were significantly increased in the hyperammonemic rats, as compared with the controls ( $\mathrm{P}<0.05$; Fig. 1A-D), which indicated the occurrence of hepatic injury.

Hyperammonemia induces hepatic injury. In the control group, the liver appeared to have a normal histological structure; the majority of hepatic cells were mononuclear and were arranged in rows that radiated out from the center (Fig. 2A). Conversely, in the hyperammonemic group, there were no signs of necrosis or inflammatory cell infiltration. In addition, there were irregular hepatic sinusoids and edema of a number of hepatocytes within the hepatic lobule and portal areas, particularly around the central vein (Fig. 2B).

Hyperammonemia induces apoptosis of hepatic cells. A TUNEL assay was used to evaluate cell apoptosis. Apoptotic cells were observed in the hyperammonemic group, but not in control group (Fig. 3A). In the hyperammonemic group, apoptotic cells were scattered within the hepatic portal and lobule areas. Quantitative analysis of TUNEL-positive cells showed that the number of apoptotic cells in the hyperammonemic group was higher than in the control group; with a significantly higher apoptotic index calculated for the hyperammonemic group $(5.84 \pm 2.25 \%)$, as compared with the control group $(1.45 \pm 0.68 \%$; P $<0.01$; Fig. 3B).

Hyperammonemia induces $S$ phase cell cycle arrest. Cell cycle distribution was analyzed using FCM. The proportion of hyperammonemic hepatic cells in the S phase increased from 36.7 to 

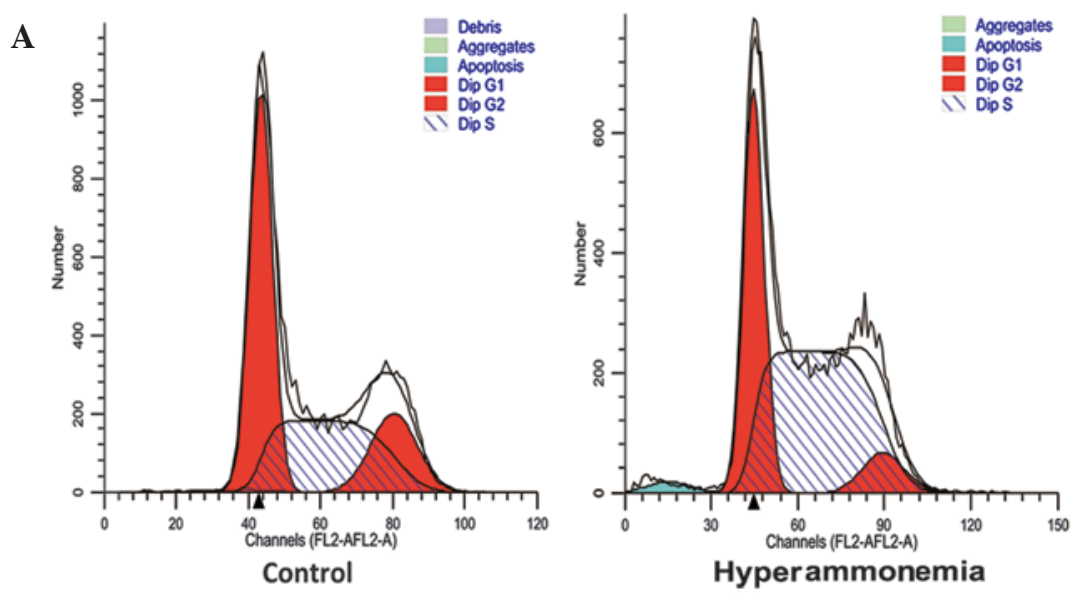

$\mathbf{B}$

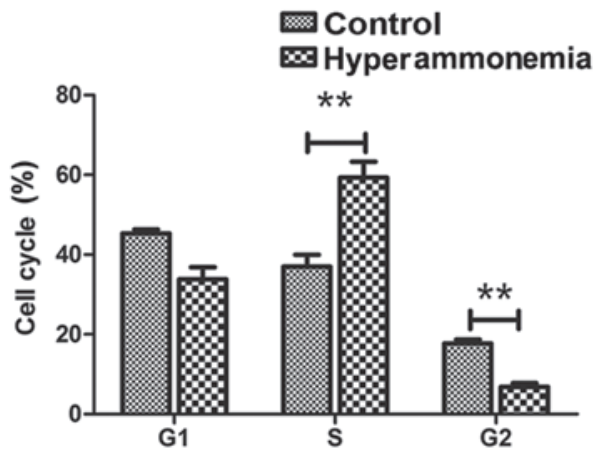

Figure 4. Cell cycle analysis. (A) Flow cytometry analysis of control and hyperammonemic group cell cycles. (B) Quantified flow cytometry results, indicating the proportion of cells in the G1, S and G2 phases of the cell cycle. ${ }^{* *} \mathrm{P}<0.01$ vs. the control. Data are presented as the mean \pm standard deviation and are representative of three independent experiments with similar results.
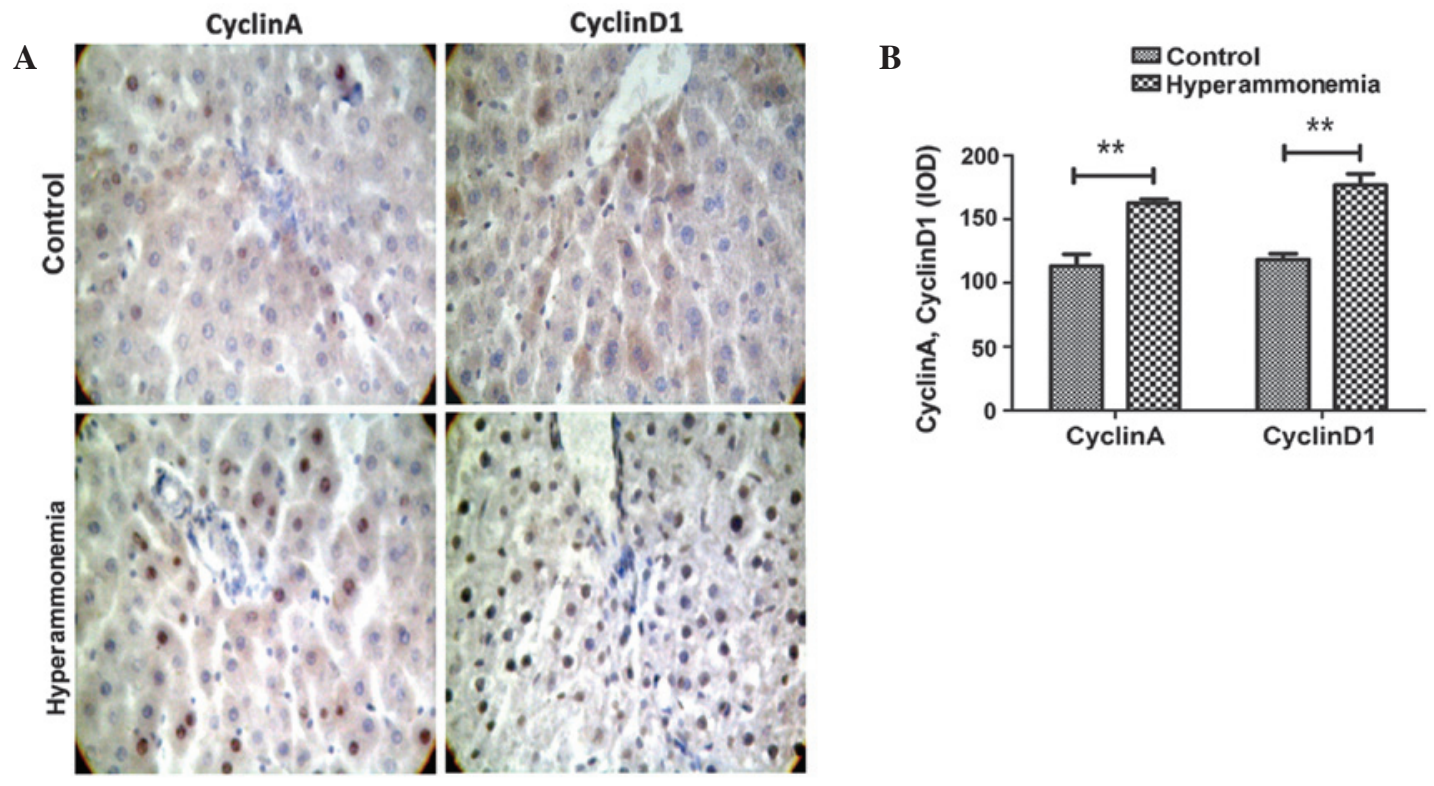

Figure 5. (A) Hyperammonaemia induced enhanced expression of cyclin A and D1, as shown by yellow chromatin in the nuclei. (3,3'-diaminobenzidine tetrahydrochloride staining; magnification, $\mathrm{x} 400$ ). (B) Cyclin A and D1 expression in hyperammonemic and control groups as measured by IOD in each tissue. ${ }^{* *} \mathrm{P}<0.01$ vs. the control. Data are presented as the mean \pm standard deviation. IOD, integrated optical density.

$59.3 \%$, those in the sub- $\mathrm{G}_{1}$ phase (apoptotic cells) increased from 0.68 to $1.89 \%$, those in the $\mathrm{G}_{0} / \mathrm{G}_{1}$ phase did not change, and the proportion of cells in the $G_{2}$ phase decreased from 17.7 to
$6.9 \%$ (Fig. 4A). Thus, hyperammonemia significantly increased the number of cells in the $\mathrm{S}$ phase and significantly decreased the number of cells in the $G_{2} / M$ phase ( $P<0.01$; Fig. $4 B$ ). 
A

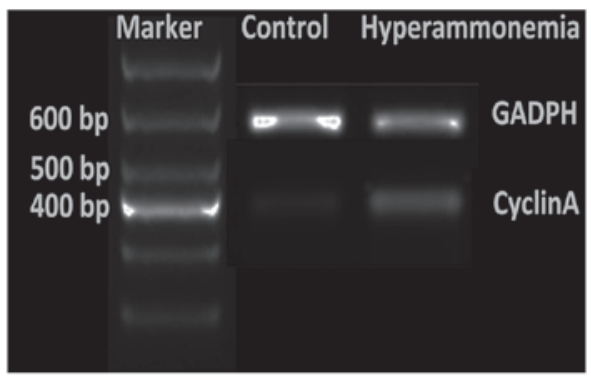

C

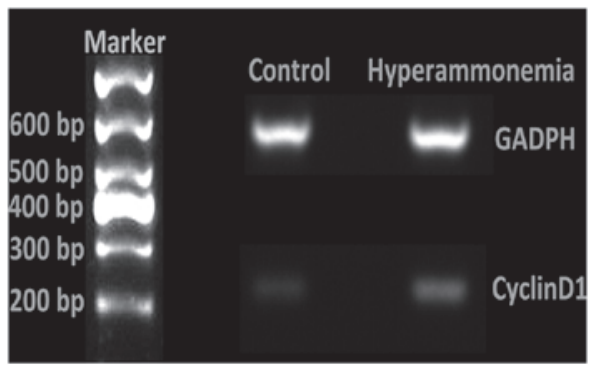

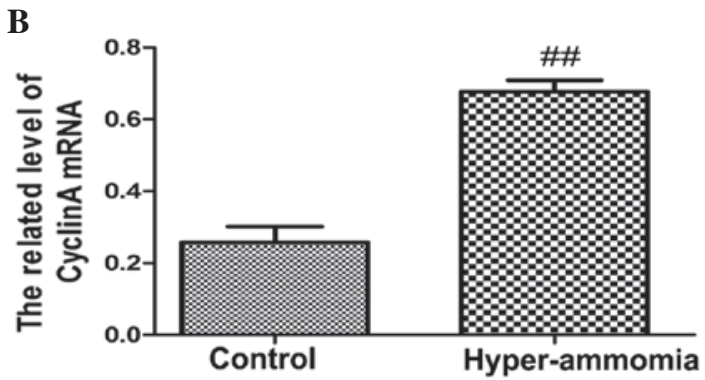

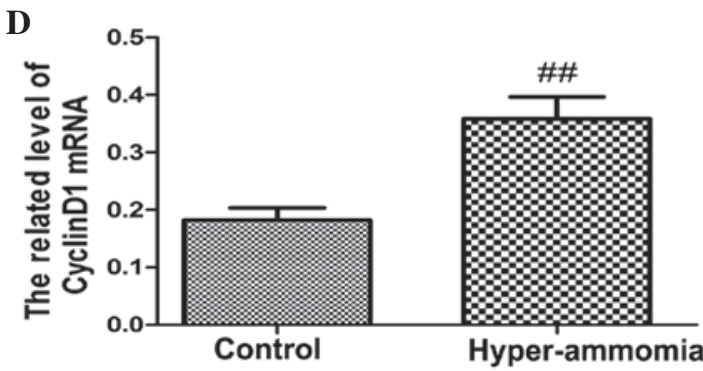

Figure 6. Analysis of hepatic cyclin A and D1 mRNA expression levels. Reverse transcription-polymerase chain reaction analyses were performed to detect the mRNA expression levels of (A and B) cyclin A and (C and D) cyclin D1. Data are presented as the mean \pm standard deviation of three independent experiments. ${ }^{\# \#} \mathrm{P}<0.01$ vs. the control. GAPDH, glyceraldehyde-3-phosphate dehydrogenase.

A

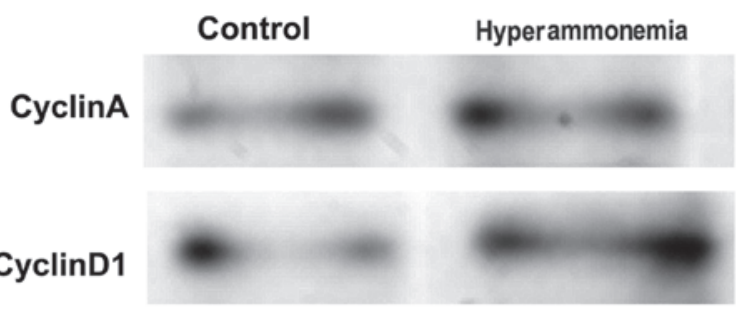

$\beta$-actin
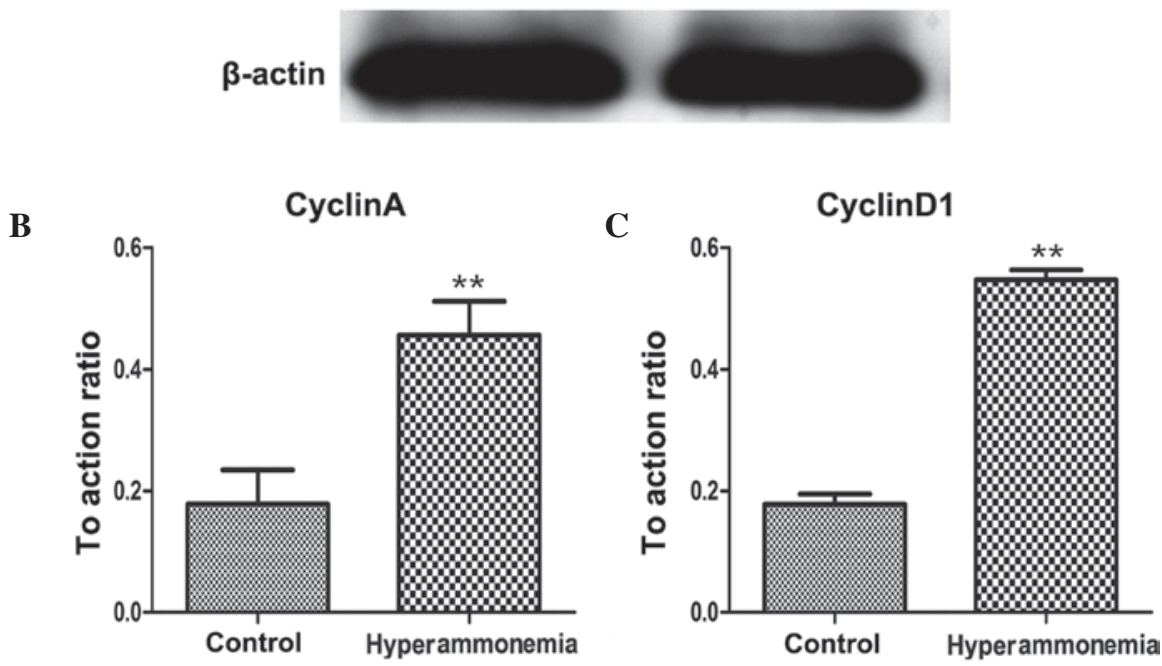

Figure 7. Analysis of hepatic cyclin A and D1 protein expression levels. (A) Western blot displaying hyperammonemia-induced protein expression levels of cyclin A and D1 in the liver. Anti- $\beta$-actin antibodies were used to ensure equal protein loading. The bands were quantified by densitometry and normalized to actin intensity. The relative expressions of cleaved (B) cyclin A and (C) cyclin D1 are shown. ${ }^{* *} \mathrm{P}<0.01$ vs. the control. Data are presented as the mean \pm standard deviation of two independent experiments.

Hyperammonemia induces an increase in the expression levels of cyclins A and D1. As compared with the controls, an increased number of cells were stained positive for cyclin A and D1 in the hyperammonemic group (Fig. 5A). The integrated optical density values for cells staining positive for cyclin A and D1 significantly increased from 113.2 and 118.3 to 162.6 and 176.9 , respectively, as compared with the controls $(\mathrm{P}<0.01$; Fig. 5B).

RT-qPCR (Fig. 6A-D) and western blotting (Fig. 7A-D) detected significantly increased mRNA and protein expression levels of cyclin A and cyclin D1, respectively, in the livers 
of rats subjected to hyperammonemia, as compared with the control group $(\mathrm{P}<0.01)$.

\section{Discussion}

Hyperammonemia is primarily caused by disorders of the urea cycle, and it is the most common symptom of HF (2,4,19-22). Hyperammonemia has been identified as a possible cause of hepatic encephalopathy following hepatic injury, where an excessive quantity of ammonia is metabolized by astrocytes, causing cell and brain swelling in vivo $(11,22,23)$. In support of this, exposing primary astrocytes to ammonia in vitro has been documented to cause cell swelling and a loss of cell viability at high ammonia concentrations (2,24-26). The findings of the present study support previous reports demonstrating the damaging effects of hyperammonemia on hepatic function $(4,14,15,27)$.

The present study measured changes in liver histology in rats that had been treated with ammonia, as compared with controls. Similar to a previous study (15), histological changes, including mild hydropic degeneration, were detected in hyperammonemic livers; however there was no presence of hepatic cell necrosis or inflammatory cell infiltration. In addition to this, it was demonstrated that hyperammonemia may cause hepatic injury by inducing hepatic cell apoptosis, which suggested that ammonia may target hepatic cells directly. Cell cycle arrest is closely associated with apoptosis (28-30), and the present study demonstrated that hyperammonemia significantly increased the number of cells in the $\mathrm{S}$ phase and significantly decreased the number of cells in the $G_{2} / M$ phase. These results suggested that hyperammonemia arrests cells in the $\mathrm{S}$ phase, which ultimately leads to a depletion of $\mathrm{G}_{2} / \mathrm{M}$ phase cells.

Cyclin A and D1 have been reported to have an important role in various cell processes (31-34). For example, previous studies have demonstrated that cyclin A-associated kinase mediates hypoxia-induced apoptosis in cardiomyocytes $(31,35,36)$. Other findings indicated that cyclin D1 regulates cell progression through the $\mathrm{G}_{1} / \mathrm{S}$ phase transition of the cell cycle, and is a key factor in tumorigenesis (37-39). The present study demonstrated that levels of cyclin A and D1 were increased in hyperammonemic hepatic cells. In order to explore the underlying mechanism of hyperammonemia-induced hepatic injury, the expression levels of cyclin A and D1 were measured in association with apoptotic signaling pathways, and it was demonstrated that hyperammonemia enhanced the mRNA and protein expression levels of cyclin A and D1. Furthermore, the present study demonstrated that BA values were elevated in hyperammonemic rats. As hyperammonemia is a consequence of severe HR, BA levels may be elevated due to the decreased number of functional hepatocytes in HR and, therefore, the ability of the liver to detoxify ammonia is compromised (2,21-23). However, the results of the present study suggested that hyperammonemia-induced hepatic cell damage may occur as a result of apoptosis signaling pathways being activated and altering cell cycle progression. Until now, there have not been a great number of studies published regarding the role of hyperammonemia in hepatic cell apoptosis and cell cycle arrest $(1,4,20)$. Therefore, the present study may offer novel insights into hyperammonemia-induced hepatic injury.
In conclusion, the results of the present study suggested that hyperammonemia-induced hepatic injury in rats may be caused by changes in the expression levels of cyclin A and D1, cell cycle arrest and cellular apoptosis. The present study also concludes that hyperammonemia appeared to be a causative factor of HF. These findings indicate possible mechanisms for hepatic injury caused by hyperammonemia, and may provide potential targets for treating or preventing further hepatic damage caused by hyperammonemia, in particular, hepatic encephalopathy.

\section{Acknowledgements}

The authors would like to thank Dr Zujiang Yu for support and excellent technical assistance during the experiments and writing.

\section{References}

1. Adeva MM, Souto G, Blanco N and Donapetry C: Ammonium metabolism in humans. Metabolism 61: 1495-1511, 2012.

2. Wright G, Noiret L, Olde Damink SW and Jalan R: Interorgan ammonia metabolism in liver failure: The basis of current and future therapies. Liver Int 31: 163-175, 2011.

3. Jones EA and Mullen KD: Theories of the pathogenesis of hepatic encephalopathy. Clin Liver Dis 16: 7-26, 2012.

4. Lee WM: Acute liver failure. N Engl J Med 329: 1862-1872, 1993.

5. Blackmore L and Bernal W: Acute liver failure. Clin Med 15: 468-472, 2015

6. Butterworth RF: Hepatic encephalopathy: A central neuroinflammatory disorder? Hepatology 53: 1372-1376, 2011.

7. Trautwein $\mathrm{C}$ and Koch A: Mechanisms of acute liver failure. In: Liver Immunology. Gershwin MR, Vierling J and Manns MP (eds). 2nd edition. Springer International Publishing, New York, NY, pp373-388, 2014.

8. Pratap Mouli V, Benjamin J, Bhushan Singh M, Mani K, Garg SK, Saraya A and Joshi YK: Effect of probiotic VSL\#3 in the treatment of minimal hepatic encephalopathy: A non-inferiority randomized controlled trial. Hepatol Res 45: 880-889, 2015.

9. Jover-Cobos M, Khetan V and Jalan R: Treatment of hyperammonemia in liver failure. Curr Opin Clin Nutr Metab Care 17: 105-110, 2014.

10. Lange CM, Bojunga J, Hofmann WP, Wunder K, Mihm U, Zeuzem S and Sarrazin C: Severe lactic acidosis during treatment of chronic hepatitis B with entecavir in patients with impaired liver function. Hepatology 50: 2001-2006, 2009.

11. Fuhrmann V, Drolz A, Jaeger B, Wewalka M, Saxa R, Horvatits T, Perkmann T, Zauner C and Ferenci P: Prognostic relevance of arterial ammonia levels in different acute and acute-on-chronic liver diseases. Crit Care 16 (Suppl 1): P390, 2012.

12. Liu CP and Yu ZJ: Study on L-Ornithine-L-Aspartate in the treatment of acute-on-chronic liver failure. Zhonghua Gan Zang Bing Za Zhi 19: 63-64, 2011 (In Chinese).

13. Schmid M,Peck-Radosavljevic M, König F, Mittermaier C, Gangl A and Ferenci P: A double-blind, randomized, placebo-controlled trial of intravenous L-ornithine-L-aspartate on postural control in patients with cirrhosis. Liver Int 30: 574-582, 2010.

14. Yu ZJ, Sun R, Liu XR, Yan JY, Gao XJ and Kan QC: Hyperammonemia-induced hepatic injury in rats: Characterization of a new animal model. Zhonghua Gan Zang Bing Za Zhi 21: 467-472, 2013 (In Chinese).

15. Li J, Yu Z, Wang Q, Li D, Jia B, Zhou Y, Ye Y, Shen S, Wang Y, $\mathrm{Li} \mathrm{S}$, et al: Hyperammonia induces specific liver injury through an intrinsic $\mathrm{Ca}^{2+}$-independent apoptosis pathway. BMC Gastroenterol 14: 151, 2014

16. Zhu Q, Gu L, Wang Y, Jia L, Zhao Z, Peng S and Lei L: The role of alpha-1 and alpha-2 adrenoceptors in restraint stress-induced liver injury in mice. PLoS One 9: e92125, 2014.

17. Bartek J and Lukas J: Mammalian G1- and S-phase checkpoints in response to DNA damage. Curr Opin Cell Biol 13: 738-747, 2001.

18. Myklebust MP, Li Z, Tran TH, Rui H, Knudsen ES, Elsaleh H, Fluge $\varnothing$, Vonen B, Myrvold HE, Leh S, et al: Expression of cyclin D1a and D1b as predictive factors for treatment response in colorectal cancer. Br J Cancer 107: 1684-1691, 2012. 
19. Degli Esposti D, Hamelin J, Bosselut N, Saffroy R, Sebagh M Pommier A, Martel C and Lemoine A: Mitochondrial roles and cytoprotection in chronic liver injury. Biochem Res Int 2012: 387626, 2012.

20. Jalan R, Gines P, Olson JC, Mookerjee RP, Moreau R, Garcia-Tsao G, Arroyo V and Kamath PS: Acute-on chronic liver failure. J Hepatol 57: 1336-1348, 2012.

21. Sarin SK, Kumar A, Almeida JA, Chawla YK, Fan ST, Garg H, de Silva HJ, Hamid SS, Jalan R, Komolmit P, et al: Acute-on-chronic liver failure: Consensus recommendations of the Asian Pacific Association for the study of the liver (APASL). Hepatol Int 3: 269-282, 2009.

22. Aldridge DR, Tranah EJ and Shawcross DL: Pathogenesis of hepatic encephalopathy: Role of ammonia and systemic inflammation. J Clin Exp Hepatol 5 (Suppl 1): S7-S20, 2015.

23. Bobermin LD, Quincozes-Santos A, Guerra MC, Leite MC, Souza DO, Gonçalves CA and Gottfried C: Resveratrol prevents ammonia toxicity in astroglial cells. PLoS One 7: e52164, 2012.

24. Rose CF: Ammonia: More than a neurotoxin? Liver Int 34 649-651, 2014.

25. Wang Q, Wang Y, Yu Z, Li D, Jia B, Li J, Guan K, Zhou Y, Chen Y and Kan Q: Ammonia-induced energy disorders interfere with bilirubin metabolism in hepatocytes. Arch Biochem Biophys 555-556: 16-22, 2014.

26. Zhang LJ, Qi R, Zhong J, Xu Q, Zheng G and Lu GM: The effect of hepatic encephalopathy, hepatic failure, and portosystemic shunt on brain volume of cirrhotic patients: A voxel-based morphometry study. PLoS One 7: e42824, 2012.

27. Jia B, Yu ZJ, Duan ZF, Lü XQ, Li JJ, Liu XR, Sun R, Gao XJ, Wang YF, Yan JY and Kan QC: Hyperammonaemia induces hepatic injury with alteration of gene expression profiles. Liver Int 34: 748-758, 2014.

28. Johnson DG and Walker CL: Cyclins and cell cycle checkpoints. Annu Rev Pharmacol Toxicol 39: 295-312, 1999.

29. Hartwell LH and Kastan MB: Cell cycle control and cancer. Science 266: 1821-1828, 1994
30. Stewart ZA, Mays D and Pietenpol JA: Defective G1-S cell cycle checkpoint function sensitizes cells to microtubule inhibitor-induced apoptosis. Cancer Res 59: 3831-3837, 1999.

31. Chibazakura T, Kamachi K, Ohara M, Tane S, Yoshikawa H and Roberts JM: Cyclin A promotes S-phase entry via interaction with the replication licensing factor Mcm7. Mol Cell Biol 31: 248-255, 2011.

32. Kang DS, Hong K-M, Park J and Bae CD: Cyclin A regulates a cell-cycle-dependent expression of CKAP2 through phosphorylation of Sp1. Biochem Biophys Res Commun 420: 822-827, 2012.

33. Pardo FS, Su M and Borek C: Cyclin D1 induced apoptosis maintains the integrity of the G1/S checkpoint following ionizing radiation irradiation. Somat Cell Mol Genet 22: 135-144, 1996.

34. Ha SY, Kim HK, Im JS, Cho HY, Chung DH and An J: Expression of Cyclin A, B1, D1, D3, and E in non-small lung cancers. J Lung Cancer 11: 33-37, 2012.

35. Meng FJ, Jiao SM and Yu B: Picroside II protects cardiomyocytes from hypoxia/reoxygenation-induced apoptosis by activating the PI3K/Akt and CREB pathways. Int J Mol Med 30: 263-270, 2012.

36. Wu D, Jiang H, Chen S and Zhang H: Inhibition of microRNA-101 attenuates hypoxia/reoxygenation-induced apoptosis through induction of autophagy in H9c2 cardiomyocytes. Mol Med Rep 11: 3988-3994, 2015.

37. Hashimoto T, Yanaihara N, Okamoto A, Nikaido T, Saito M, Takakura S, Yasuda M, Sasaki H, Ochiai K and Tanaka T: Cyclin D1 predicts the prognosis of advanced serous ovarian cancer. Exp Ther Med 2: 213-219, 2011.

38. Pontano LL and Diehl JA: Speeding through cell cycle roadblocks: Nuclear cyclin D1-dependent kinase and neoplastic transformation. Cell Div 3: 12, 2008.

39. Peurala E, Koivunen P, Haapasaari KM, Bloigu R and Jukkola-Vuorinen A: The prognostic significance and value of cyclin D1, CDK4 and p16 in human breast cancer. Breast Cancer Res 15: R5, 2013. 